\title{
Some New Inequalities for Lipschitz Functions via a Functional
}

\author{
Bir Fonksiyonel yardımı ile Lipschitz Fonksiyonları için Bazı Yeni Eşitsizlikler
}

\author{
İmdat İşCAN ${ }^{1, a}$, Cuma ALTUNSOY ${ }^{2, b}$, Mahir KADAKAL ${ }^{* 1, \mathrm{c}}$ \\ ${ }^{I}$ Giresun University, Faculty of Arts and Science, Deptartment of Mathematics, 28200, Giresun \\ ${ }^{2}$ National Education Ministry, Mustafa Kemal Secondary School, Mathematics Teacher, 28200, Giresun
}

• Geliş tarihi / Received: 25.06.2018 • Düzeltilerek geliş tarihi / Received in revised form: 28.09.2018 • Kabul tarihi / Accepted: 10.10 .2018

\begin{abstract}
This study is about getting some new integral inequalities for Lipschitz functions by using a functional defined via a Lipschitz function. Here, some new Hermite-Hadamard $(\mathrm{H}-\mathrm{H})$ type inequalities are first found out as a corollary of main theorems. Afterwards, some new H-H type inequalities for Lipschitz functions by means of inequalities which are used for p-convex functions are obtained.
\end{abstract}

Keywords: Convex function, Lipschitz function,p-Convex function

$\ddot{O} z$

Bu çalışma, bir Lipschitz fonksiyonu yardımı ile tanımlanmış bir fonksiyonel kullanarak Lipschitz fonksiyonları için bazı yeni integral eşitsizliklerin elde edilmesi ile ilgilidir. Burada ilk önce, bazı yeni Hermite-Hadamard tipi eşitsizlikler, ana teoremlerin bir sonucu olarak ortaya çıkarllacaktır. Daha sonra ise, p-konveks fonksiyonlar için kullanılan eşitsizlikler aracılı̆̆lyla Lipschitz fonksiyonları için yeni Hermite Hadamard tipi eşitsizlikler elde edilecektir.

Anahtar kelimeler: Konveks fonksiyon, Lipschitz fonksiyonu,p-konveks fonksiyon

\footnotetext{
*c Mahir KADAKAL; mahirkadakal@gmail.com; Tel: (0454) 31052 65; orcid.org/0000-0002-0240-918X

${ }^{a}$ orcid.org/0000-0001-6749-0591 ${ }^{\mathrm{b}}$ orcid.org/0000-0003-1865-9842
} 


\section{Preliminaries and Fundamentals}

Definition 1.1. (Pečarić, 1992) A function $f: I \subseteq \mathbb{R} \rightarrow \mathbb{R}$ is said to be convex if the following inequality

$f(\lambda u+(1-\lambda) v) \leq \lambda f(u)+(1-\lambda) f(v)$

holds for all $u, v \in I$ and $\lambda \in[0,1]$. If the above inequality reverses, then the $f$ is said to be concave on the interval $I \neq \emptyset$.

The most important integral inequalities for convex functions are the Hermite-Hadamard inequalities. The following double integral inequalities are well known as the HermiteHadamard inequalities in the literature.

Theorem 1.2. Given $f:[u, v] \rightarrow \mathbb{R}$ be a convex function. Then the following inequalities

$f\left(\frac{u+v}{2}\right) \leq \frac{1}{v-u} \int_{u}^{v} f(x) d x \leq \frac{f(u)+f(v)}{2}$

are known as the $\mathrm{H}-\mathrm{H}$ type inequalities.

Some refinements of the $\mathrm{H}-\mathrm{H}$ inequalities have been extensively studied by a number of authors (Hadamard, 1893; Dragomir and Pearce, 2002; Dragomir, 2002) and they have obtained some new refinements of the $\mathrm{H}-\mathrm{H}$ inequalities.

Definition 1.3. (İşcan, 2014) Given $I \subset \mathbb{R} \backslash\{0\}$ be a real interval. $f: I \rightarrow \mathbb{R}$ is called harmonically convex function, if

$f\left(\frac{u v}{\lambda u+(1-\lambda) v}\right) \leq \lambda f(v)+(1-\lambda) f(u)$

for all $u, v \in I$ and $\lambda \in[0,1]$. If the above inequality is reversed, then $f$ is called harmonically concave function.

Definition 1.4. (İşcan, 2016) Given $I \subset(0, \infty)$ be a real interval and $p \in \mathbb{R} \backslash\{0\} . f: I \rightarrow \mathbb{R}$ is called a $p$-convex function, if

$f\left(\left[\lambda u^{p}+(1-\lambda) v^{p}\right]^{\frac{1}{p}}\right) \leq \lambda f(u)+(1-\lambda) f(v)$

for all $u, v \in I$ and $\lambda \in[0,1]$. If this inequality is reversed, then the function $f$ is said to be $p$ concave.

From this definition, we can easily see that for $p=1$ and $p=-1, p$-convexity reduces to ordinary convexity and harmonically convexity of functions defined on the interval $I \subset(0, \infty)$, respectively.

$\mathrm{H}-\mathrm{H}$ type inequalities for the $p$-convex function are following:

Theorem 1.5. (İşcan, 2016) Given $f: I \subset$ $(0, \infty) \rightarrow \mathbb{R}$ be a $p$-convex function, $p \in \mathbb{R} \backslash\{0\}$, $u, v \in I, u<v$. If $f \in L[u, v]$, then we obtain

$$
\begin{aligned}
f\left(\left[\frac{u^{p}+v^{p}}{2}\right]^{\frac{1}{p}}\right) & \leq \frac{p}{v^{p}-u^{p}} \int_{u}^{v} \frac{f(x)}{x^{1-p}} d x \\
& \leq \frac{f(u)+f(v)}{2} .
\end{aligned}
$$

These inequalities are sharp (Kunt and İşcan, 2017a,b,c). $f$ is said to be $p$-concave, if these inequalities are reversed. We refer the reader to the recent papers related to $p$-convexity (Kunt and İşcan, 2017d; Latif et al, 2015; Niculescu, 2000; Yang and Tseng, 1999) and references therein.

Definition 1.6. (Roberts and Varberg, 1973) $f: I \rightarrow \mathbb{R}$ is said to satisfy the Lipschitz condition if there is a constant $M>0$ such that

$|f(u)-f(v)| \leq M|u-v|, \quad \forall u, v \in I$.

Theorem 1.7. (Roberts and Varberg, 1973) If the function $f: I \rightarrow \mathbb{R}$ is convex, then $f$ satisfies a Lipschitz condition on any closed interval $[a, b]$ contained in the interior $I^{\circ}$ of $I$. Consequently, the function $f$ is absolutely continuous on the interval $[a, b]$ and continuous on $I^{\circ}$.

Theorem 1.8. (Dragomir et al, 2000) Given $f: I \subset(0, \infty) \rightarrow \mathbb{R}$ be an $M$-Lipschitzian mapping on $I$ and $\alpha, \beta \in I, \alpha<\beta . H$ is defined on the interval $[0,1]$ by

$H(t)=\frac{1}{\beta-\alpha} \int_{\alpha}^{\beta} f\left(t x+(1-t) \frac{\alpha+\beta}{2}\right) d x$

is a Lipschitzian function for all $t \in[0,1] . H(t)$ is an $\left(\frac{M(\beta-\alpha)}{4}\right)$-Lipschitzian function.

Theorem 1.9. (Dragomir et al, 2000) Given $f: I \subset(0, \infty) \rightarrow \mathbb{R}$ be an $M$-Lipschitzian mapping on the interval $I$ and $\alpha, \beta \in I, \alpha<\beta$. Then 
$\left|\frac{f\left(t \beta+(1-t) \frac{\alpha+\beta}{2}\right)+f\left(t \alpha+(1-t) \frac{\alpha+\beta}{2}\right)}{2}-H(t)\right|$

$\leq \frac{M t}{3}(\beta-\alpha)$

for all $t \in[0,1]$.

In (İşcan et al., 2018), the authors established the following $\mathrm{H}-\mathrm{H}$ type inequalities for Lipschitzian functions.

Theorem 1.10. Given $f: I \subset(0, \infty) \rightarrow \mathbb{R}$ be an $M$ Lipschitzian function on the interval $I$ of real numbers and $\alpha, \beta \in I, \alpha<\beta$. Then following inequalities hold for $p \geq 1$ :

i)

$$
\begin{aligned}
& \left|\frac{f(\alpha)+f(\beta)}{2}-\frac{p}{\beta^{p}-\alpha^{p}} \int_{\alpha}^{\beta} \frac{f(x)}{x^{1-p}} d x\right| \\
& \leq 2 M\left|\beta^{p}-\alpha^{p}\right|^{\frac{1}{p}} \frac{p^{2}}{(p+1)(2 p+1)}
\end{aligned}
$$

ii)

$$
\begin{aligned}
& \left|\frac{p}{\beta^{p}-\alpha^{p}} \int_{\alpha}^{\beta} \frac{f(x)}{x^{1-p}} d x-f\left(\left[\frac{\alpha^{p}+\beta^{p}}{2}\right]^{\frac{1}{p}}\right)\right| \\
& \leq 2 M\left(\frac{1}{2}\right)^{\frac{1}{p}}\left|\beta^{p}-\alpha^{p}\right|^{\frac{1}{p}} \frac{p}{p+1} .
\end{aligned}
$$

\section{Main Results}

Theorem 2.1. Given $f: I \subset(0, \infty) \rightarrow \mathbb{R}$ be an $M$ Lipschitzian function on the interval $I, a, b \in I$, $a<b$. For $p \geq 1$, the function defined by

$H_{p}(t)$

$=\frac{p}{b^{p}-a^{p}} \int_{a}^{b} \frac{f\left(\left[t x^{p}+(1-t) \frac{a^{p}+b^{p}}{2}\right]^{\frac{1}{p}}\right)}{x^{1-p}} d x$,

fulfills the following Hölder condition

$\left|H_{p}\left(t_{2}\right)-H_{p}\left(t_{1}\right)\right| \leq \frac{p M}{p+1}\left(\frac{b^{p}-a^{p}}{2}\right)^{\frac{1}{p}}\left|t_{2}-t_{1}\right|^{\frac{1}{p}}$

for $t_{1}, t_{2} \in[0,1]$.

Proof. Let $t_{1}, t_{2} \in[0,1]$. We have the following inequality:

$$
\begin{aligned}
& \left|H_{p}\left(t_{2}\right)-H_{p}\left(t_{1}\right)\right| \\
& =\frac{p}{b^{p}-a^{p}} \mid \int_{a}^{b} \frac{f\left(\left[t_{2} x^{p}+\left(1-t_{2}\right)\left(\frac{a^{p}+b^{p}}{2}\right)\right]^{\frac{1}{p}}\right)}{x^{1-p}} d x \\
& -\int_{a}^{b} \frac{f\left(\left[t_{1} x^{p}+\left(1-t_{1}\right)\left(\frac{a^{p}+b^{p}}{2}\right)\right]^{\frac{1}{p}}\right)}{x^{1-p}} d x \mid \\
& \leq \frac{p M}{b^{p}-a^{p}} \mid d x . \\
& \times \int_{a}^{b \mid} \mid\left[\frac{\left.t_{2} x^{p}+\left(1-t_{2}\right) \frac{a^{p}+b^{p}}{2}\right]^{\frac{1}{p}}-\left[t_{1} x^{p}+\left(1-t_{1}\right) \frac{a^{p}+b^{p}}{2}\right]^{\frac{1}{p}}}{x^{1-p}} \mid\right.
\end{aligned}
$$

Set $A_{p}:=\frac{a^{p}+b^{p}}{2}$. For $0 \leq x<y$ and $0<\alpha \leq 1$ if we use the following well known inequality,

$y^{\alpha}-x^{\alpha} \leq(y-x)^{\alpha}$

we have

$$
\begin{aligned}
& \left|H_{p}\left(t_{1}\right)-H_{p}\left(t_{2}\right)\right| \leq \frac{p M}{b^{p}-a^{p}} \\
& \times \int_{a}^{b}\left|\frac{\left[t_{2} x^{p}+\left(1-t_{2}\right) A_{p}\right]^{\frac{1}{p}}-\left[t_{1} x^{p}+\left(1-t_{1}\right) A_{p}\right]^{\frac{1}{p}}}{x^{1-p}}\right| d x \\
& \leq \frac{p M}{b^{p}-a^{p}} \int_{a}^{b} \frac{\left|t_{2} x^{p}+\left(1-t_{2}\right) A_{p}-\left(t_{1} x^{p}+\left(1-t_{1}\right) A_{p}\right)\right|^{\frac{1}{p}}}{x^{1-p}} d x \\
& =\frac{p M}{b^{p}-a^{p}}\left|t_{2}-t_{1}\right|^{\frac{1}{p}} \int_{a}^{b} \frac{\left|x^{p}-A_{p}\right|^{\frac{1}{p}}}{x^{1-p}} d x .
\end{aligned}
$$

Now, let us calculate the integral in inequality (3). Since

$$
x^{p}-A_{p}=0 \Rightarrow x=M_{p}=\left(\frac{a^{p}+b^{p}}{2}\right)^{\frac{1}{p}}
$$

One can write the following equality:

$$
\begin{aligned}
& \int_{a}^{b} \frac{\left|x^{p}-A_{p}\right|^{\frac{1}{p}}}{x^{1-p}} d x \\
& =\int_{a}^{M_{p}} \frac{\left(A_{p}-x^{p}\right)^{\frac{1}{p}}}{x^{1-p}} d x+\int_{M_{p}}^{b} \frac{\left(x^{p}-A_{p}\right)^{\frac{1}{p}}}{x^{1-p}} d x
\end{aligned}
$$

It is easily seen that 


$$
\begin{aligned}
& \int_{a}^{M_{p}} \frac{\left(A_{p}-x^{p}\right)^{\frac{1}{p}}}{x^{1-p}} d x \\
& =\int_{M_{p}}^{b} \frac{\left(x^{p}-A_{p}\right)^{\frac{1}{p}}}{x^{1-p}} d x \\
& =\frac{\left(\frac{b^{p}-a^{p}}{2}\right)^{\frac{p+1}{p}}}{p+1}
\end{aligned}
$$

Finally, if we put equality (5) in inequality (3), we obtain the desired result:

$\left|H_{p}\left(t_{2}\right)-H_{p}\left(t_{1}\right)\right| \leq \frac{p M}{p+1}\left(\frac{b^{p}-a^{p}}{2}\right)^{\frac{1}{p}}\left|t_{2}-t_{1}\right|^{\frac{1}{p}}$

Corollary 2.2. For $p=1$, the inequality

$\left|H_{1}\left(t_{2}\right)-H_{1}\left(t_{1}\right)\right| \leq M \frac{b-a}{4}\left|t_{2}-t_{1}\right|$

holds. This coincides with the Theorem 1.8.

Theorem 2.3. Given the function $f: I \subset(0, \infty) \rightarrow$ $\mathbb{R} M$-Lipschitzian on the interval $I, a, b \in I$, $a<b$ and $p \geq 1$. Then, the following inequality

$$
\begin{array}{|l}
\mid \frac{f\left(\left[t a^{p}+(1-t)\left(\frac{a^{p}+b^{p}}{2}\right)\right]^{\frac{1}{p}}\right)}{2} \\
+\frac{f\left(\left[t b^{p}+(1-t)\left(\frac{a^{p}+b^{p}}{2}\right)\right]^{\frac{1}{p}}\right)}{2}-H_{p}(t) \mid
\end{array}
$$

$\leq 2 M t^{\frac{1}{p}}\left|b^{p}-a^{p}\right|^{\frac{1}{p}} \frac{p^{2}}{(p+1)(2 p+1)}$ inequality holds for all $t \in[0,1]$.

\section{Proof. Let}

$u=\left[t a^{p}+(1-t)\left(\frac{a^{p}+b^{p}}{2}\right)\right]^{\frac{1}{p}}$

and

$$
v=\left[t b^{p}+(1-t)\left(\frac{a^{p}+b^{p}}{2}\right)\right]^{\frac{1}{p}}
$$

If we make the changes of variable $z=\left[t x^{p}+(1-t)\left(\frac{a^{p}+b^{p}}{2}\right)\right]^{\frac{1}{p}}$

with $\frac{a^{p}+b^{p}}{2}=A_{p}$ in the equality

$H_{p}(t)=\frac{p}{b^{p}-a^{p}} \int_{a}^{b} \frac{f\left(\left[t x^{p}+(1-t) A_{p}\right]^{\frac{1}{p}}\right)}{x^{1-p}} d x$

then we have

$$
\begin{aligned}
& H_{p}(t) \\
& =\frac{p}{t\left(b^{p}-a^{p}\right)} \int_{a}^{b} \frac{f\left(\left[t x^{p}+(1-t) A_{p}\right]^{\frac{1}{p}}\right)}{x^{1-p}} t d x \\
& =\frac{p}{v^{p}-u^{p}} \int_{u}^{v} \frac{f(z)}{z^{1-p}} d z .
\end{aligned}
$$

Now, using inequality (1) with $\alpha=\left[t a^{p}+(1-\right.$ t) $\left.A_{p}\right]^{\frac{1}{p}}=u, \quad \beta=\left[t b^{p}+(1-t) A_{p}\right]^{\frac{1}{p}}=v \quad$ and $v^{p}-u^{p}=t\left(b^{p}-a^{p}\right)$, we obtain the following inequality:

$$
\begin{aligned}
& \left|\frac{f(u)+f(v)}{2}-\frac{p}{v^{p}-u^{p}} \int_{u}^{v} \frac{f(z)}{z^{1-p}} d z\right| \\
& \leq 2 M t^{\frac{1}{p}}\left|b^{p}-a^{p}\right|^{\frac{1}{p}} \frac{p^{2}}{(p+1)(2 p+1)} .
\end{aligned}
$$

Corollary 2.4. The results obtained for $p=1$ coincides with Theorem 1.9.

Proposition 2.5. Given $f: I \subset(0, \infty) \rightarrow \mathbb{R}$ a $p$ convex on $I$ and $\alpha, \beta \in I, \alpha<\beta$. Then, for all $t \in[0,1]$, the following inequalities

i)

$$
\begin{aligned}
& H_{p}(t)=\frac{p}{\beta^{p}-\alpha^{p}} \int_{\alpha}^{\beta} \frac{f\left(\left[t x^{p}+(1-t)\left(\frac{\alpha^{p}+\beta^{p}}{2}\right)\right]^{\frac{1}{p}}\right)}{x^{1-p}} d x \\
& \leq \frac{p}{\beta^{p}-\alpha^{p}} \int_{\alpha}^{\beta} \frac{f(x)}{x^{1-p}} d x,
\end{aligned}
$$

ii)

$$
\begin{aligned}
& H_{p}(t)=\frac{p}{\beta^{p}-\alpha^{p}} \int_{\alpha}^{\beta} \frac{f\left(\left[t x^{p}+(1-t)\left(\frac{\alpha^{p}+\beta^{p}}{2}\right)\right]^{\frac{1}{p}}\right)}{x^{1-p}} d x \\
& \geq f\left(M_{p}\right)
\end{aligned}
$$


and

$$
\begin{aligned}
& f\left(M_{p}\right) \leq H_{p}(t) \\
& =\frac{p}{\beta^{p}-\alpha^{p}} \int_{\alpha}^{\beta} \frac{f\left(\left[t x^{p}+(1-t)\left(\frac{\alpha^{p}+\beta^{p}}{2}\right)\right]^{\frac{1}{p}}\right)}{x^{1-p}} d x \\
& \leq \frac{f\left(\left[t \alpha^{p}+(1-t)\left(\frac{\alpha^{p}+\beta^{p}}{2}\right)\right]^{\frac{1}{p}}\right)}{2} \\
& -\frac{-f\left(\left[t \beta^{p}+(1-t)\left(\frac{\alpha^{p}+\beta^{p}}{2}\right)\right]^{\frac{1}{p}}\right)}{2}
\end{aligned}
$$

hold with $M_{p}=\left(\frac{\alpha^{p}+\beta^{p}}{2}\right)^{\frac{1}{p}}$.

Proof. (i) Since $f$ is a $p$-convex function, we have

$$
\begin{aligned}
& H_{p}(t) \\
& =\frac{p}{\beta^{p}-\alpha^{p}} \int_{\alpha}^{\beta} \frac{f\left(\left[t x^{p}+(1-t)\left(\frac{\alpha^{p}+\beta^{p}}{2}\right)\right]^{\frac{1}{p}}\right)}{x^{1-p}} d x \\
& \leq \frac{p}{\beta^{p}-\alpha^{p}} \int_{\alpha}^{\beta}\left[\frac{t f(x)}{x^{1-p}}+\frac{(1-t) f\left(M_{p}\right)}{x^{1-p}}\right] d x \\
& =\frac{p}{\beta^{p}-\alpha^{p}}\left[t \int_{\alpha}^{\beta} \frac{f(x)}{x^{1-p}} d x+(1-t) f\left(M_{p}\right) \int_{\alpha}^{\beta} \frac{1}{x^{1-p}} d x\right] \\
& =\frac{p}{\beta^{p}-\alpha^{p}}\left[t \int_{\alpha}^{\beta} \frac{f(x)}{x^{1-p}} d x+(1-t) f\left(M_{p}\right) \frac{\beta^{p}-\alpha^{p}}{p}\right] .
\end{aligned}
$$

By using the $\mathrm{H}-\mathrm{H}$ inequality for $p$-convex functions, we get the following inequality:

$$
\begin{aligned}
H_{p}(t) & \leq t \frac{p}{\beta^{p}-\alpha^{p}} \int_{\alpha}^{\beta} \frac{f(x)}{x^{1-p}} d x \\
& +(1-t) \frac{p}{\beta^{p}-\alpha^{p}} \int_{\alpha}^{\beta} \frac{f(x)}{x^{1-p}} d x \\
& =\frac{p}{\beta^{p}-\alpha^{p}} \int_{\alpha}^{\beta} \frac{f(x)}{x^{1-p}} d x .
\end{aligned}
$$

(ii) Let

$u=\left[t \beta^{p}+(1-t)\left(\frac{\alpha^{p}+\beta^{p}}{2}\right)\right]^{\frac{1}{p}}$

and

$v=\left[t \alpha^{p}+(1-t)\left(\frac{\alpha^{p}+\beta^{p}}{2}\right)\right]^{\frac{1}{p}}$.

If we make the change of variable

$$
z=\left[t \alpha^{p}+(1-t)\left(\frac{\alpha^{p}+\beta^{p}}{2}\right)\right]^{\frac{1}{p}}
$$

with $\frac{\alpha^{p}+\beta^{p}}{2}=A_{p}$ in the equality

$$
\begin{aligned}
& H_{p}(t) \\
& =\frac{p}{\beta^{p}-\alpha^{p}} \int_{\alpha}^{\beta} \frac{f\left(\left[t x^{p}+(1-t)\left(\frac{\alpha^{p}+\beta^{p}}{2}\right)\right]^{\frac{1}{p}}\right)}{x^{1-p}} d x,
\end{aligned}
$$

then we have

$H_{p}(t)=\frac{p}{v^{p}-u^{p}} \int_{u}^{v} \frac{f(z)}{z^{1-p}} d z$.

By using the $\mathrm{H}-\mathrm{H}$ inequality for $p$-convex function

$$
\begin{aligned}
H_{p}(t) & =\frac{p}{v^{p}-u^{p}} \int_{u}^{v} \frac{f(z)}{z^{1-p}} d z \\
& \geq f\left(\left[\frac{u^{p}+v^{p}}{2}\right]^{\frac{1}{p}}\right) \\
& =\left(\left[\frac{\alpha^{p}+\beta^{p}}{2}\right]^{\frac{1}{p}}\right) \\
& =f\left(M_{p}\right)
\end{aligned}
$$

is obtained. Consequently one gets

$f\left(M_{p}\right) \leq \frac{p}{v^{p}-u^{p}} \int_{u}^{v} \frac{f(z)}{z^{1-p}} d z \leq \frac{f(u)+f(v)}{2}$

which is equivalent inequality (6).

\section{References}

Dragomir, S.S., Cho, Y.J. and Kim, S.S., 2000. Inequalities of Hadamard's type for Lipschitzian mappings and their applications. Journal of Mathematical Analysis and Applications, 245, 2, 489-501.

Dragomir, S.S., 2002. On Some New inequalities of Hermite-Hadamrd Type for m-Convex Functions, Tamkang. J. of Math., 33(1), 45-55.

Dragomir, S.S., Pearce, C.E.M., 2002. Selected Topics on Hermite-Hadamard Inequalities and Applications, RGMIA Monograph, Victoria University, online: http://rgmia.org/monographs.php 
Hadamard, J., 1893. Etude sur les proprietes des fonctions entieres en particulier d'une fonction consideree par Riemann. J. Math. Pures Appl. $58,171-215$.

İscan, İ., 2014. Hermite-Hadamard type inequalities for harmonically convex functions. Hacettepe Journal of Mathematics and Statistics, 43(6), 935-942.

İşcan, İ., 2016. Ostrowski type inequalities for pconvex functions, New Trends in Mathematical Sciences, NTMSCI 4, No. 3, 140-150.

İşcan, İ., Altunsoy, C. and Kadakal, M., 2018. New inequalities on Lipschitz functions, International Conference on Mathematics and Mathematics Education, Ordu University, Ordu, 27-29 Haziran, Book of abstracts, s.169.

Kunt, M. and İşcan, İ., 2017a. On new HermiteHadamard-Fejer type inequalities for p-convex functions via fractional integrals, Communication in Mathematical Modeling and Applications, 2(1), 1-15.

Kunt, M. and İşcan, İ., 2017b. Hermite-HadamardFejer type inequalities for p-convex functions, Arab J. Math. Sci., 23(2), 215-230.

Kunt, M. and İşcan, İ., 2017c. Hermite-HadamardFejer type inequalities for $\mathrm{p}$-convex functions via fractional integrals. Iranian Journal of Science and Technology, Transactions A: Science, Doi: 10.1007/s40995-017-0352-4

Kunt, M. and İşcan, İ., 2017d. Hermite-Hadamard type inequalities for p-convex functions via fractional integrals. Moroccan J. Pure Appl. Anal., 3(1), 22-35.

Latif, M.A. Dragomir S. S. and Momaniat, E., 2015. Some Fejer type integral inequalities for geometrically-arithmetically-convex functions with applications, RGMIA Research Report Collection, 18, Article 25, 18 pp.

Niculescu, C.P., 2000. Convexity according to the geometric mean. Math. Inequal. Appl., 3(2), 155-167.

Pečarić, J., Proschan, F. and Tong, Y. L., 1992. Convex Functions, Partial Orderings and Statistical Applications. Academic Press, Inc., 469 pp, Boston.

Roberts, A.W. and Varberg, D.E., 1973. Convex Functions. Academic Press, 300 pp, New York.

Yang, G.S. and Tseng, K.L., 1999. On certain integral inequalities related to Hermite-Hadamard inequalities. J. Math. Anal. Appl., 239, 180-187. 